\title{
Development of Cryogenic Techniques for Characterizing Energy Storage Materials in Electrochemical Process
}

\author{
Minghao Zhang and Shirley Meng \\ UCSD, La Jolla, California, United States
}

Lithium-ion batteries (LIBs) commercially dominate portable energy storage and have been extended to hybrid/electric vehicles by utilizing electrode materials with enhanced energy density. However, the energy density and cycling life of LIBs must extend beyond the current reach of commercial electrodes to meet the performance requirements for transportation applications [1]. Carbon-based anodes, serving as the main negative electrodes in LIBs, have an intrinsic capacity limitation due to the intercalation mechanism [2]. Recently, researchers proposed to use Li metal as the anode to achieve higher energy density [3]. However, the dendritic growth of Li metal during cycling will result in low coulombic efficiency as well as safety issue which is detrimental for practical applications. It is proposed solid state electrolytes (SSE) are compatible with lithium metal because of lithium penetration resistance [4], which can be the ultimate choice to achieve the energy density of $500 \mathrm{Wh} / \mathrm{kg}$.

The performance of existing all-solid-state batteries is still not optimal, which is mainly attributed to the poor interfacial stability. Further improvements and developments of these energy storage materials rely on a fundamental understanding of their electrochemical cycling mechanisms at atomic level. Characterization of $\mathrm{Li}$ anodes and SSE/Li-metal interfaces remains difficult due to their sensitivity to beam damage, the nano-scale of interfacial decomposition products, and their buried nature [5]. Our group has demonstrated the importance of cryogenic techniques for preparation (cryogenic focused ion beam, cryo-FIB) and characterization (cryogenic transmission electron microscopy, cryo-TEM), to preserve the morphology and structure of lithium metal [6,7]. These breakthroughs enabled unprecedented characterization of plated Li metal and are currently being extended to all-solid-state batteries.

The first demonstration of the functionality of the cryo-TEM will focus on characterizing the morphology and crystallinity of electrochemically cycled lithium metal with different electrolytes. As the Coulombic efficiency of $\mathrm{Li}$ metal varies greatly with electrolyte properties, we compared two representative electrolytes, a high-concentration electrolyte (HCE) and a commercial carbonate electrolyte (CCE). It is found Sheet-like inactive Li appears in the HCE sample (Figure 1a), whereas inactive Li in the CCE retains a whisker-like morphology (Figure 1e). Based on the (110) lattice plane distance of body-centered cubic $\mathrm{Li}$, the region that contains crystalline metallic $\mathrm{Li}^{0}$ is highlighted in green in the high-resolution TEM (HRTEM) images for both electrolytes (Figure $1 \mathrm{~b}$ and $\mathrm{f}$ ). Compared with the inactive Li obtained from $\mathrm{CCE}$, a much smaller area of metallic $\mathrm{Li}^{0}$ component is observed in HCE. This indicates that most of the deposited metallic $\mathrm{Li}^{0}$ in $\mathrm{HCE}$ has been successfully stripped, corresponding to the high Coulombic efficiency.

This work will also cover recent advances on cryo-EM development for Li/LiPON interface. The combination of cryo-FIB and cryo-EM is necessary for quantitative structural and chemical analysis due to extreme susceptibility of both lithium metal and LiPON to air and beam damage. We discover the chemical evolution along the Li/LiPON interface and $\mathrm{N}$ species diffusion into Li metal region. EELS results show a gradient phosphorus and oxygen, into the Li metal. The obtained results provide new insights of decomposition, diffusion of chemical species, and chemical evolution along the Li/LiPON interface, which leads to a better understanding of the stability. 

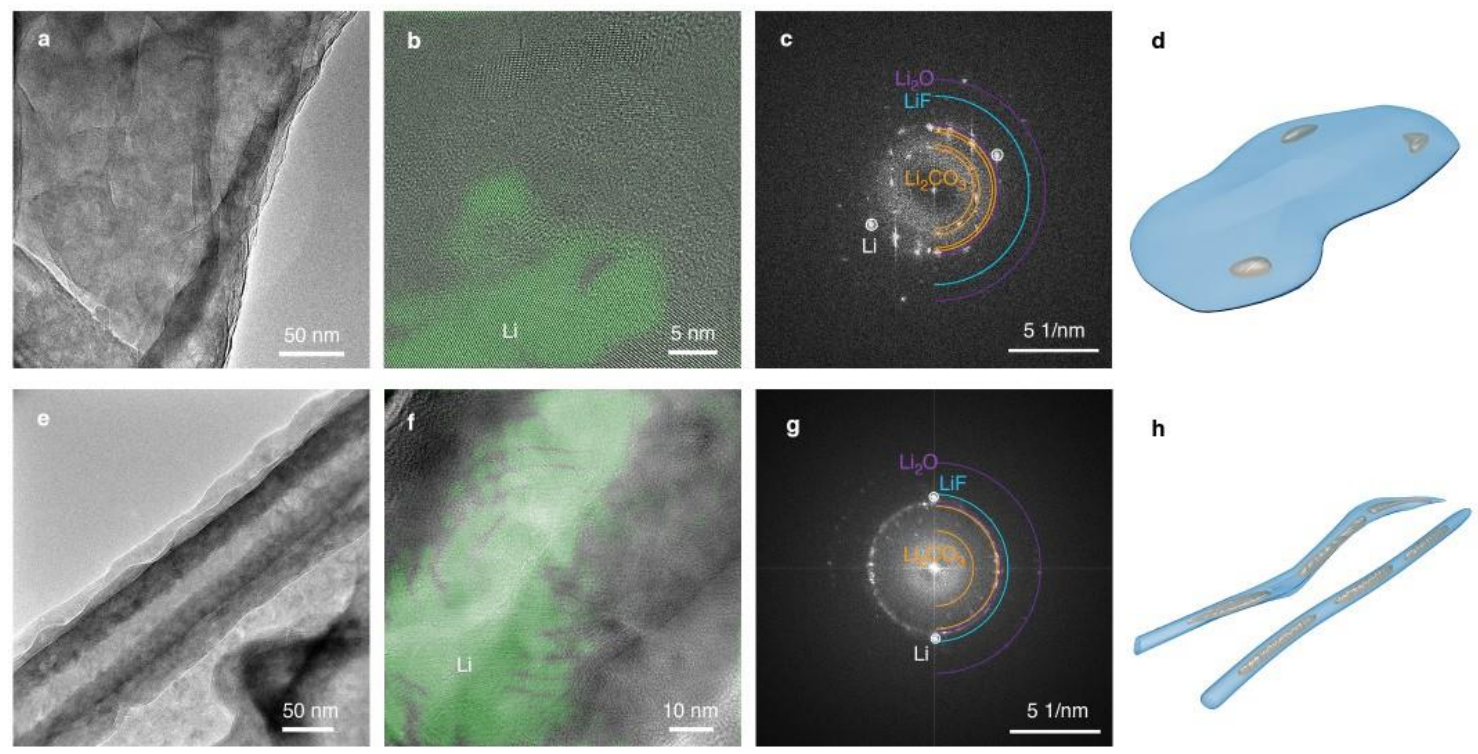

h

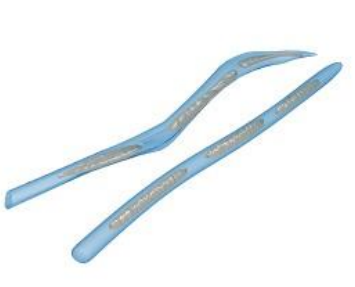

Figure 1. Nanostructures of inactive Li generated in HCE (a-d) and CCE (e-h) by Cryo-TEM [8].

\section{References}

[1] M. Armand and J.-M. Tarascon, Nature 451 (2008), 652.

[2] P. Poizot, S. Laruelle, S. Grugeon, L. Dupont and J. Tarascon, Nature 407 (2000), 496.

[3] X.-B. Cheng, R. Zhang, C.-Z. Zhao, F. Wei, and J.-G. Zhang, Advanced Science 3 (3) (2016), 1500213.

[4] K. Kerman, A. Luntz, V. Viswanathan, Y.-M. Chiang, and Z. Chen, Journal of the Electrochemical Society 164 (7) (2017), A1731.

[5] X. Wang, Y. Li, and Y. S. Meng, Joule 2 (2018) 1-10.

[6] J. Z. Lee, T. A. Wynn, M. A. Schroeder, J. Alvarado, X. Wang, K. Xu, and Y. S. Meng, ACS Energy Letters 4 (2) (2019), 489.

[7] X. Wang, M. Zhang, J. Alvarado, S. Wang, M. Sina, B. Lu, J. Bouwer, W. Xu, J. Xiao, J.-G. Zhang, and Y. S. Meng, Nano Letters 17 (12) (2017), 7606.

[8] C. Fang, J. Li, M. Zhang, Y. Zhang, F. Yang, J. Z. Lee, M. Lee, J. Alvarado, M. A. Schroeder, Y. Yang, B. Lu, N. Williams, M. Ceja, L. Yang, M. Cai, J. Gu, K. Xu, X. Wang, and Y. S. Meng, Nature 572 (2019) 511.

The authors gratefully acknowledge funding support from the U.S. Department of Energy, Office of Basic Energy Sciences, under Award Number DE-SC0002357 (program manager Dr. Jane Zhu). 